\title{
BMJ Open Association of metabolic syndrome with risk of cardiovascular disease mortality and all-cause mortality among Malaysian adults: a retrospective cohort study
}

Kee Chee Cheong (10), ${ }^{1}$ Kuang Hock Lim (1) , ${ }^{2}$ Sumarni Mohd Ghazali, ${ }^{2}$ Chien Huey Teh, ${ }^{2}$ Yong Kang Cheah, ${ }^{3}$ Azli Baharudin, ${ }^{4}$ Hui Li Lim, ${ }^{5}$ Abdul Muneer Abdul Hamid, ${ }^{1}$ Feisul Idzwan Mustapha, ${ }^{6}$ Mohd Azahadi Omar ${ }^{1}$

To cite: Chee Cheong K, Lim KH, Ghazali SM, et al. Association of metabolic syndrome with risk of cardiovascular disease mortality and all-cause mortality among Malaysian adults: a retrospective cohort study. BMJ Open 2021;11:e047849. doi:10.1136/ bmjopen-2020-047849

- Prepublication history and additional supplemental material for this paper are available online. To view these files, please visit the journal online (http://dx.doi.org/10.1136/ bmjopen-2020-047849).

Received 17 December 2020 Accepted 03 August 2021

Check for updates

(C) Author(s) (or their employer(s)) 2021. Re-use permitted under CC BY-NC. No commercial re-use. See rights and permissions. Published by BMJ.

For numbered affiliations see end of article.

Correspondence to Mr Kee Chee Cheong; kee@moh.gov.my

\section{ABSTRACT}

Objectives This study is aimed at determining the association between metabolic syndrome and risk of cardiovascular disease (CVD) mortality and all-cause mortality among Malaysian adults.

Design Retrospective cohort study.

Setting The Malaysian Non-Communicable Disease Surveillance (MyNCDS-1) 2005/2006.

Participants A total of 2525 adults (1013 men and 1512 women), aged 24-64 years, who participated in the MyNCDS-1 2005/2006.

Methods Participants' anthropometric indices, blood pressure, fasting lipid profile and fasting blood glucose levels were evaluated to determine the prevalence of metabolic syndrome by the Harmonized criteria. Participants' mortality status were followed up for 13 years from 2006 to 2018. Mortality data were obtained via record linkage with the Malaysian National Registration Department. The Cox proportional hazards regression model was applied to determine association between metabolic syndrome (MetS) and risk of CVD mortality and all-cause mortality with adjustment for selected sociodemographic and lifestyle behavioural factors.

Results The overall point prevalence of MetS was $30.6 \%$ (95\% Cl: 28.0 to 33.3). Total follow-up time was 31668 person-years with 213 deaths (111 (11.3\%) in MetS subjects and 102 (6.1\%) in non-MetS subjects) from all-causes, and 50 deaths (33 (2.9\%) in MetS group and 17 (1.2\%) in non-MetS group) from CVD. Metabolic syndrome was associated with a significantly increased hazard of CVD mortality (adjusted HR: 2.18 (95\% Cl: 1.03 to 4.61), $\mathrm{p}=0.041$ ) and all-cause mortality (adjusted HR: 1.47 (95\% Cl: 1.00 to 2.14), $\mathrm{p}=0.048$ ). These associations remained significant after excluding mortalities in the first 2 years.

Conclusions Our study shows that individuals with MetS have a higher hazard of death from all-causes and CVD compared with those without MetS. It is thus imperative to prescribe individuals with MetS, a lifestyle intervention along with pharmacological intervention to improve the individual components of MetS and reduce this risk.
Strengths and limitations of this study

- This study data came from a nationally representative, population-based study with large sample size that makes the findings generalisable to the noninstitutionalised population of Malaysia.

- We controlled for potential confounders including age, gender, education level, physical activity, fruit and vegetable intake, smoking and alcohol consumption in the Cox regression models.

- There were deaths that were not medically certified.

\section{INTRODUCTION}

Metabolic syndrome (MetS) refers to the clustering of metabolic abnormalities that is linked to higher risk of cardiovascular disease (CVD), type 2 diabetes, cancer mortality, ${ }^{12}$ non-alcoholic fatty liver disease ${ }^{3}$ and all-cause mortality. ${ }^{2}{ }^{4-6}$ These metabolic abnormalities include generalised obesity/abdominal obesity, glucose intolerance, elevated blood pressure and dyslipidaemia. ${ }^{7}$ Increasing rates of MetS across Asia-Pacific countries are raising concern that it is becoming a public health threat that requires urgent intervention. $^{8}$

Up to around 20 years ago, prevalence of MetS in Asian countries was low compared with Western countries. ${ }^{9}$ Subsequently, changes in lifestyle, namely poor dietary habits, increased refined sugar consumption and inadequate dietary fibre intake coupled with lower physical activity levels, have resulted in an increasing trend of MetS in Asian countries. ${ }^{8}$ Furthermore, Asians tend to have a higher percentage of visceral fat compared with Westerners with the same body mass index (BMI). ${ }^{10-12}$ Since abdominal obesity is a component of MetS, Asians are thus more 
likely to have MetS. Given the current trend, MetS will likely become more prevalent in Asian countries than in Western countries. ${ }^{8}$ Previous studies have indicated that MetS is an independent risk factor of all-cause mortality and CVD-related mortality. ${ }^{613} 14$ However, prevalence of MetS and its components may have different impacts on CVD mortality risk in different ethnic groups and countries. ${ }^{15}$ In Malaysia, the top three causes of death in 2019 were ischaemic heart diseases (15\%), pneumonia (12.2\%) and CVDs $(8 \%) .{ }^{16}$ Data on risk of mortality related to MetS remain unavailable. Most of the studies were confined to estimating MetS prevalence (ranging from 32\% to 42\%) and its associated factors among Malaysian adults. ${ }^{17-21}$ National data on the risk of CVD and all-cause mortality associated with MetS can provide insight into the burden of this condition that are essential for public health programme managers and clinicians in managing MetS. Therefore, we conducted a retrospective cohort study using data from the first Malaysian Non-Communicable Disease Surveillance (MyNCDS-1) 2005/2006 as baseline data on MetS and lifestyle risk factors. ${ }^{22}$ Participants of the MyNCDS-1 2005/2006 were followed up prospectively to assess the association between MetS and all-cause and CVD mortality.

\section{METHODS}

\section{Study population}

We used a retrospective cohort study design using data from the MyNCDS-1 2005/2006. MyNCDS-1 was a nationwide, cross-sectional, population-based baseline survey on non-communicable diseases and its risk factors conducted in 2005-2006. ${ }^{22}$ A minimum sample size of 2533 was calculated based on prevalence of obesity of $5 \%$, precision of $1.2 \%$ and design effect of 2. After adding $20 \%$ expected non-response, the final target sample size was 3040. Participants were selected from 13 states and 1 federal territory (Kuala Lumpur) in Malaysia by multistage, cluster sampling using the year 2000 National Household Sampling Frame with the assistance of the Malaysian Department of Statistics. Stratifying variables were state and setting (urban/rural), with enumeration blocks, living quarters and households as the primary, secondary and elementary sampling units, respectively. A sample weight variable was created for strata (state and setting) and cluster (enumeration block and living quarters) for use in the analysis to generate population estimates. There was no oversampling of any particular groups in this study. The number of enumeration blocks and living quarters selected per state was based on the desired sample size and proportionate to the size of the 2005 Malaysian adult (age 25-64 years) population of each state. In all, a total of 398 enumeration blocks and 1683 living quarters were selected. All members of households in the selected living quarters who met the eligibility criteria were included in the sample. The inclusion criteria were Malaysian citizens, between 25 and 64 years of age. Excluded from the study were pregnant women, and mentally ill, very ill, or institutionalised individuals. Participation in the study was voluntary and informed consent was obtained. Eligible individuals who refused to participate and those who could not be reached after three attempts were considered as non-responders. The final sample comprised of 2572 subjects after removing the non-responders yielding a response rate of $84.6 \%$. Data collection was carried out from September 2005 to February 2006, with a total of 2572 adults participating in the survey. The details of the methodology and results of MyNCDS-1 have been described elsewhere. ${ }^{22}$

\section{Estimation of minimum sample size required for this study}

Sample size for this study was calculated using Power and Sample Size Calculation V.3.1.6. ${ }^{23}$ A previous study estimated $2.8 \%$ CVD mortality among non-MetS subjects. ${ }^{6}$ If the estimated HR of CVD mortality for individuals with MetS compared with non-MetS was 2.0, we needed to study 805 MetS subjects and 805 non-MetS subjects to be able to reject the null hypothesis that the HR equals 1 with $80 \%$ power. The type 1 error probability $(\alpha)$ associated with this hypothesis test was 0.05 . We used the uncorrected $\chi^{2}$ statistic to evaluate this null hypothesis. ${ }^{23}$

\section{Baseline data}

Baseline data extracted from the MyNCDS-1 2005/2006 survey were participants' sociodemographic characteristics (gender, ethnicity and age), lifestyle factors (physical activity, smoking status and alcohol consumption), anthropomorphic measurements (height, weight and waist circumference (WC)), blood pressure and biochemical measurements (overnight fasting venous blood of lipid profile and glucose).

\section{Assessment of lifestyle risk factors}

Current smokers were those who smoked any tobacco product either daily (smokes a tobacco product at least once a day) or occasionally (smokes but not every day). ${ }^{24}$ Current drinkers were those who consumed alcohol in the past 12 months. ${ }^{25}$ We dichotomised alcohol consumption as yes/no alcohol consumption in the past 12 months because of poor or incomplete response on the frequency and quantity of alcohol consumption. Intensity and frequency of physical activity were measured using the Global Physical Activity Questionnaire V.2.0. ${ }^{26}$ Physical activity was quantified in metabolic equivalent (MET) minutes per week (MET min/week). For work and recreation, 1 min of moderate intensity activity merited four METs, while $1 \mathrm{~min}$ of vigorous intensity activity merited eight METs. Cycling and walking merited four MET scores each. 'Physically active' was defined by a total score of at least $600 \mathrm{MET} \mathrm{min} /$ week. Adequate fruit and vegetable intake was defined as consumption of $\geq 5$ servings of fruits and vegetables daily.

\section{Anthropometric and blood pressure measurements}

Height was measured without footwear to the nearest $0.1 \mathrm{~cm}$ using a stadiometer. Weight was measured to the nearest $0.1 \mathrm{~kg}$ using a balance beam scale or Seca 
beam scale with minimal clothing and no shoes. WC was measured directly over skin or over light clothing to the nearest $0.1 \mathrm{~cm}$ at the smallest circumference below the rib cage and above the umbilicus while standing with abdominal muscles relaxed. Resting blood pressure was measured by the auscultatory method. Blood pressure was measured two times, (three times if the first two readings differed by more than $10 \mathrm{~mm} \mathrm{Hg}$ ) at no less than 30 $\mathrm{s}$ between measurements, and averaged.

\section{Biochemical measurements}

Overall, $5 \mathrm{~mL}$ of venous blood samples after overnight fasting were collected for measurement of total cholesterol, high-density lipoprotein cholesterol (HDL-C), triglycerides and glucose. All blood samples were kept on dry ice prior to laboratory analysis. Concentrations of HDL-C, triglyceride and fasting blood glucose were measured using enzymatic assay kits (Automated HDL Cholesterol Flex, Triglyceride Flex and Glucose Flex). Total cholesterol was determined using an enzymatic colorimetric method.

\section{Definition of metabolic syndrome}

There are a number of different definitions of MetS that differ by predetermined criteria and cutoffs. The most commonly used definitions are the National Cholesterol Education Program Expert Panel III (NCEP-ATP III) criteria, ${ }^{27}$ the revised NCEP-ATP III criteria, ${ }^{28}$ the International Diabetes Federation criteria (IDF) ${ }^{29}$ criteria and the 'Harmonized' criteria jointly proposed by the IDF Task Force on Epidemiology and Prevention, National Heart, Lung and Blood Institute, American Heart Association, World Heart Federation and the International Association for the Study of Obesity. ${ }^{30}$ Previous studies have shown that Asians have increased risk of CVD and mortality at lower levels of adiposity compared with Western populations. ${ }^{10} 1131$ Therefore, the 'Harmonized' criteria was chosen as it was more appropriate in identifying MetS in the Malaysian population. ${ }^{32}$ Using the 'Harmonized' criteria, MetS is defined as presence of three or more of the following: central obesity (men: $W C \geq 90 \mathrm{~cm}$, women: WC $\geq 80 \mathrm{~cm}$ ), blood pressure $\geq 130 / 85 \mathrm{~mm} \mathrm{Hg}$ or known hypertension), hyperglycaemic (fasting plasma glucose $\geq 5.6 \mathrm{mmol} / \mathrm{L}$ or known DM type 2 ), hypertriglyceridaemia $(\geq 1.7 \mathrm{mmol} / \mathrm{L})$, and reduced HDL-C (men: $<1.0$ $\mathrm{mmol} / \mathrm{L}$, women $<1.3 \mathrm{mmol} / \mathrm{L})$.

\section{Follow-up}

Participants of MyNCDS-1 were followed up for approximately 13 years from March 2006 to December 2018. Data on mortality during this period were obtained by matching the survey participants' identification number with records in the death registry administered by the National Registration Department of Malaysia. All deaths from diseases of the circulatory system coded as International Statistical Classification of Diseases and Related Health Problems, 10th revision (ICD-10) I00 to ICD-10 I99 (I00-I099 acute rheumatic fever, I05-I09 chronic rheumatic heart diseases, I10-I15 hypertensive diseases, I20-I25 ischaemic heart diseases, I26-I28 pulmonary heart disease and diseases of pulmonary circulation, I30-I52 other forms of heart disease, I60-I69 cerebrovascular diseases, I70-I79 diseases of arteries, arterioles and capillaries, I80-I89 diseases of veins, lymphatic vessels and lymph nodes, not elsewhere classified, I95-I99 other and unspecified disorders of the circulatory system) according to the ICD-10 V.2019 were classified as cardiovascular mortalities. ${ }^{33}$

\section{Statistical analyses}

All statistical analyses were performed using IBM SPSS V.18.0 for Windows (IBM Corp). The analyses took into account the complex survey design and unequal selection probabilities using the sample weight created in the complex sampling design earlier. Poststratification weights were applied in the analysis to account for nonresponse. Participants without data on identification number were excluded from analysis. The point prevalence of MetS, selected sociodemographic and health risk behaviours are presented in frequencies, percentages and 95\% CIs by living status (dead/alive). The Cox proportional hazards regression model was applied to determine the association between MetS and all-cause and CVD mortality adjusting for selected sociodemographic characteristics and health risk behaviours. Sensitivity analysis was performed, whereby Cox regression models were also generated for all-cause mortality and CVD mortality excluding mortalities in the first 2 years of follow-up. Test of proportional hazards assumptions were met $(p>0.05)$, and there were no significant two-way interactions between all the independent variables (All interactions tested, $p>0.05$ ) in the multiple Cox regression models for both all-cause and CVD deaths.

\section{Patient and public involvement}

Patients or the public were not involved in the design, or conduct, or reporting, or dissemination plans of our research.

\section{RESULTS}

Of the 2572 participants, a total of 2525 (98.2\%) participants were amenable to analysis after excluding participants missing data on identification number. The sample consisted of 1013 (40.1\%) men and 1512 (59.9\%) women. The sociodemographic characteristics, prevalence of MetS and prevalence of health risk behaviours of the participants at baseline are presented in table 1. There were $845 \mathrm{MetS}$ cases among the 2525 participants at baseline, giving an overall estimated point prevalence of MetS was $30.6 \%$. Univariable analysis showed significant associations between age group, ethnicity and individual MetS components with MetS status, but not for health risk behaviours (smoking, alcohol consumption, fruits and vegetables intake and physical activity), gender and ethnicity. During the 13 years follow-up, a total of 213 
Open access

Table 1 Baseline characteristics of participants by metabolic syndrome status $(n=2525)$

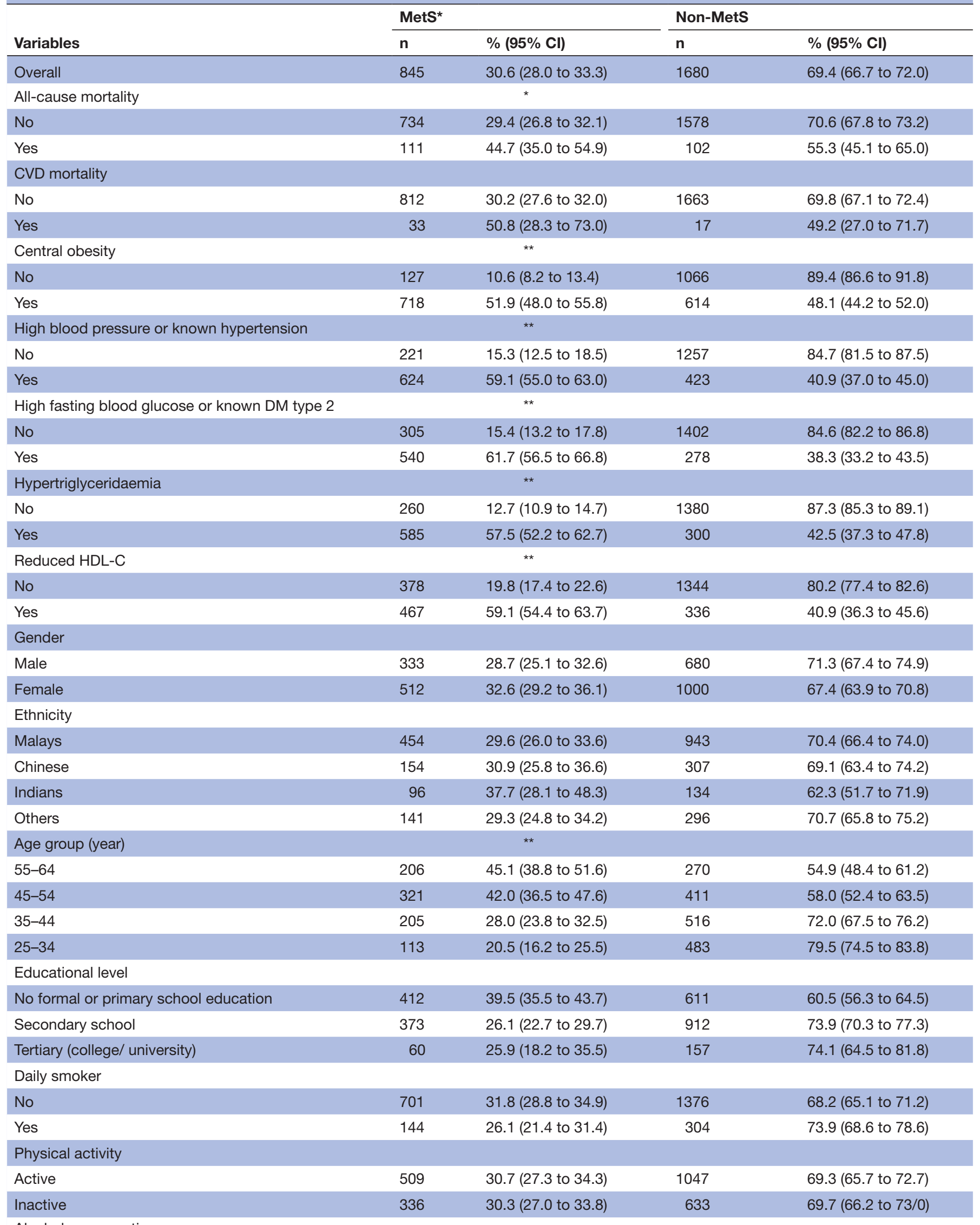

Alcohol consumption 
Table 1 Continued

\begin{tabular}{|c|c|c|c|c|}
\hline \multirow[b]{2}{*}{ Variables } & \multicolumn{2}{|c|}{ MetS* } & \multicolumn{2}{|c|}{ Non-MetS } \\
\hline & $\mathbf{n}$ & $\%(95 \% \mathrm{Cl})$ & $\mathbf{n}$ & $\%(95 \% \mathrm{Cl})$ \\
\hline No & 776 & 31.1 (28.3 to 34.0$)$ & 1497 & $68.9(66.0$ to 71.7$)$ \\
\hline \multicolumn{5}{|c|}{ Fruit and vegetable intake } \\
\hline Inadequate & 774 & 30.5 (27.8 to 33.3$)$ & 1528 & 69.5 (66.7 to 72.2$)$ \\
\hline
\end{tabular}

Pearson's $\chi^{2}$ test of independence was performed $\left({ }^{*} \mathrm{p}<0.01\right.$, $\left.{ }^{* *} \mathrm{p}<0.001\right)$.

*Based on Harmonized criteria: central obesity (men: WC $\geq 90 \mathrm{~cm}$, women: WC $\geq 80 \mathrm{~cm}$ ), blood pressure of $\geq 130 / 85 \mathrm{~mm}$ Hg or known hypertension, fasting plasma glucose $\geq 5.6 \mathrm{mmol} / \mathrm{L}$ or known DM type 2 , hypertriglyceridaemia ( $\geq 1.7 \mathrm{mmol} / \mathrm{L}$ ), Reduced HDL-C (men: $<1.0 \mathrm{mmol} / \mathrm{L}$, women $<1.3$ $\mathrm{mmol} / \mathrm{L})$.

CVD, cardiovascular disease; HDL-C, high-density lipoprotein cholesterol.

deaths (111 (11.3\%) in MetS group and $102(6.1 \%)$ in nonMetS group) from all-causes, and 50 deaths $(33(2.9 \%)$ in MetS group and 17 (1.2\%) in non-MetS group) from CVD were recorded by the National Registration Department, with total follow-up time of 31668 person-years (table 1). Baseline characteristics of participants by allcause mortality and CVD mortality are supplied in online supplemental table 1 . We performed univariate analysis to explore associations between the metabolic components and sociodemographic characteristics with all cause and CVD mortality. Central obesity, hypertension, high fasting blood glucose, hypertriglyceridaemia, reduced HDL-C, male gender, older age group, no formal/primary school education, smoker were significantly associated with allcause mortality. Whereas hypertriglyceridaemia, male gender and smoker were significantly associated with CVD mortality (online supplemental table 2).

Figure 1A,C shows unadjusted and age and sex-adjusted all-cause mortality curves, whereas figure 1B,D shows unadjusted and age and sex-adjusted CVD mortality curves; for MetS and non-MetS groups. These curves show an increase in mortality over the 13-year follow-up period.

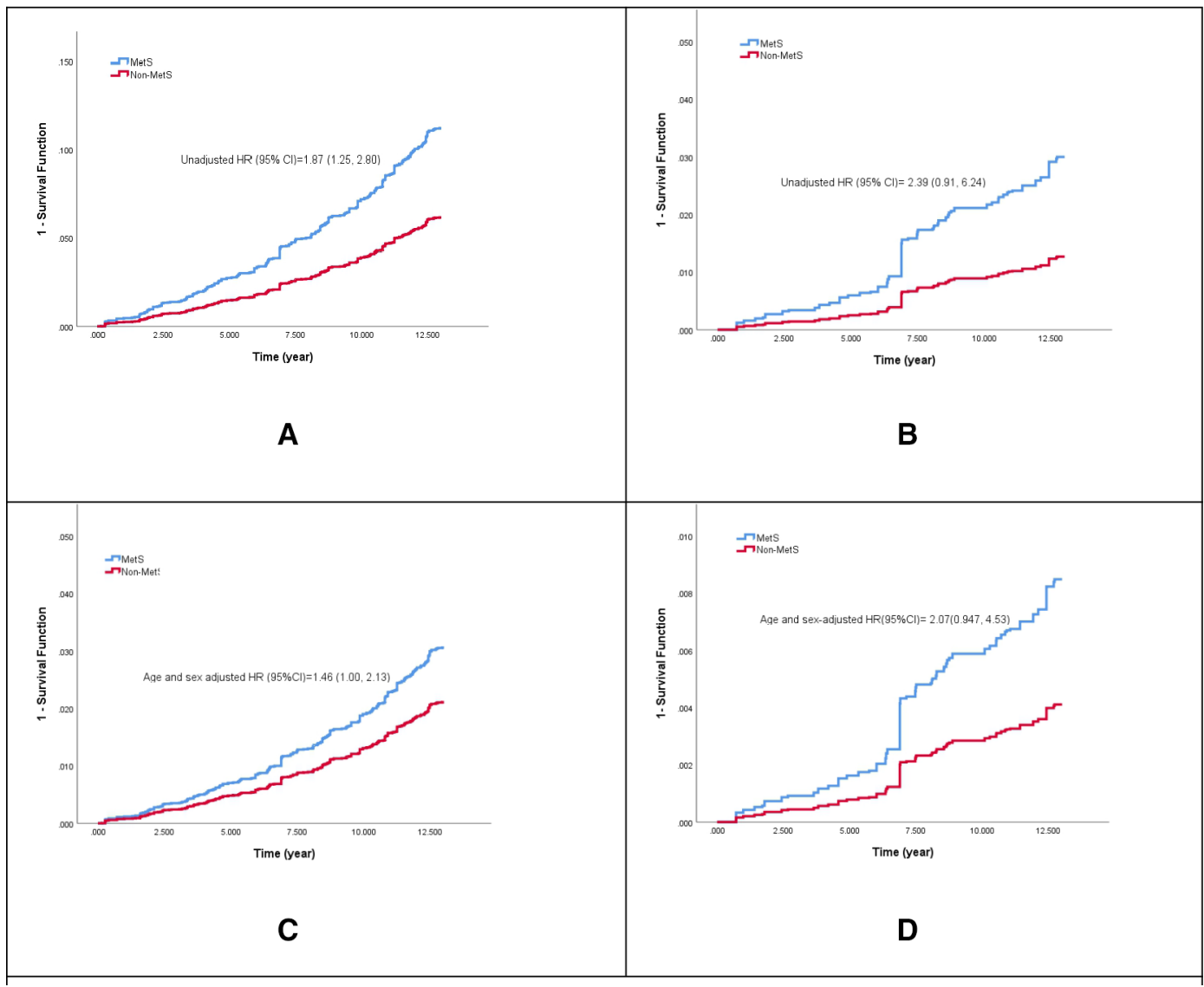

Figure 1 Comparison of mortality curves between MetS and non-MetS groups: (A) Unadjusted all-cause mortality; (B) Unadjusted CVD mortality; (C) Age and sex-adjusted all-cause mortality; and (D) Age and sex-adjusted CVD mortality. 
Table 2 The associations between metabolic syndrome and all-cause mortality and cardiovascular disease mortality among Malaysian adults population

\begin{tabular}{|c|c|c|c|}
\hline Mortality & $\begin{array}{l}\text { Unadjusted HR } \\
(95 \% \mathrm{Cl})\end{array}$ & $\begin{array}{l}\text { Age and sex-adjusted HR } \\
(95 \% \mathrm{Cl})\end{array}$ & $\begin{array}{l}\text { Multiple Cox regression, } \\
\text { adjusted HR }(95 \% \mathrm{Cl}) \dagger\end{array}$ \\
\hline \multicolumn{4}{|l|}{ Metabolic syndromeł } \\
\hline $\begin{array}{l}\text { All-cause (excluding mortalities in the first } \\
2 \text { years) }(n=2505)\end{array}$ & $2.00(1.31,3.08)^{\star *}$ & $1.59(1.07,2.37)^{\star}$ & $1.60(1.07,2.38)^{\star}$ \\
\hline
\end{tabular}

${ }^{*}$ p value $<0.05 .{ }^{* *} p$ value $<0.01 .95 \%$.

†Complex sample multiple Cox regression analysis was performed, adjusted for gender, age, education (secondary school education and above), physical activity, fruit \& vegetable intake, smoking status and alcohol consumption. Test of proportional hazard was performed for all models showed $p>0.05$. No significant two-way interactions among all the independent variables $(p>0.05)$. $\ddagger$ Based on Harmonized criteria. CVD, cardiovascular disease.

Generally, risk of all cause and CVD mortality were higher for the MetS group compared with the non-MetS group.

In the univariable analysis, MetS was significantly associated with all cause mortality (unadjusted HR: $1.87: 95 \%$ CI: 1.25 to 2.80). After adjustment for age and sex, association was marginally significant. The unadjusted and age and sex-adjusted hazard of CVD mortality was 2.4 (unadjusted HR: 2.39: 95\% CI: 0.91 to 6.24), and 2.1 (age and sex-adjusted HR: 2.07: : $95 \%$ CI: 0.95 to 4.53 ) times higher in the MetS compared with the non-MetS group, but no significant association was observed. In the multiple Cox regression models, MetS was significantly associated with increased hazard of CVD mortality (adjusted HR: 2.18, 95\% CI: 1.03 to 4.61 ) and of all-cause mortality (adjusted HR: 1.47, 95\% CI: 1.00 to 2.14) after adjusting for gender, age, education level, physical activity, fruit and vegetable intake, smoking status and alcohol consumption. Sensitivity analysis showed these associations remained significant after excluding mortalities in the first 2-year follow-up (table 2). Multiple Cox regression stratified by ethnicity was performed to assess differences in the HR by ethnicity. MetS significantly increased the hazard of all-cause mortality among Malays, but not among the other ethnic groups. No significant increase in hazard of CVD mortality was observed in MetS groups in all ethnic groups. However, these estimates were unstable due to extremely small sample sizes in certain cells and should be interpreted cautiously (online supplemental table 2).

\section{Post hoc power analysis}

We calculated post hoc power using PS software, and based on adjusted HR (relative risk) of CVD mortality of MetS subjects relative to non MetS subjects of 2.38, 845 MetS subjects, 1680 non-MetS subjects, we obtained power of $82 \%$. Therefore, the probability of detecting a significant difference between MetS and non-MetS mortality was $82 \%$. The type 1 error probability associated with this test was 0.05 .

\section{DISCUSSION}

In this study, the overall point prevalence of MetS was $30.6 \%$ based on the Harmonized criteria. The reported prevalence is in agreement with a recent review of studies conducted among Malaysian adults who reported prevalences of between $25 \%$ and $40 \%$, depending on the criteria used to define MetS. ${ }^{34}$

Our data show that presence of MetS was associated with approximately 1.5 times the hazard of all-cause mortality and twofold increase in hazard of CVD mortality. Our findings are consistent with numerous past studies. Malik et $a l^{13}$ followed up participants of the second National Health and Nutrition Examination Survey (NHANES II) 1976-1980 for an average of 13 years. Their analyses reported MetS was associated with 2.02 (95\% CI: 1.42 to 2.89) HR with respect to CHD mortality, and 1.82 (1.40-2.37) hour for CVD mortality and 1.40 (1.19-1.66) for all-cause mortality after controlling for age, gender, smoking, physical activity and total cholesterol. ${ }^{13}$ Another study which analysed data from the subsequent survey, the NHANES III, 1988-1994, with a longer follow-up period of 20 years, found MetS was associated with 1.4 times risk of mortality. ${ }^{35}$ An analysis of the more recent 1999-2010 NHANES cohort suggested that the association of MetS with all-cause mortality could be modified by BMI. ${ }^{2}$ In the study, normal- weight-MetS subjects had an HR for allcause mortality of 1.70 (1.16-2.51) while obese-MetS had an HR of 1.30 (1.07-1.60), compared with normal-weightno MetS subjects. However, for CVD mortality, only the normal-weight-MetS group had a higher hazard (HR 2.12 (95\% CI: 1.16 to 2.51 ) of CVD mortality compared with the normal-weight-no MetS group. ${ }^{2}$ A pooled analysis of 87 prospective studies predominantly in western countries, with a total of 951083 patients, reported that MetS was associated with a 2-fold increase in risk of CVD mortality and a 1.5-fold increase in risk of all-cause mortality. ${ }^{6}$ Another meta-analysis of prospective cohort 
studies in the US (six studies), Europe (eight studies) and Asia (seven studies) published from the year 2001 to December 2009 reported similar findings of approximately 1.5 -fold risk of all-cause mortality for individuals with MetS. ${ }^{36}$ However, a large prospective populationbased study, which involved Dutch people aged 50 years and above showed a relatively weak association between MetS and risk of all-cause mortality regardless of MetS definition used, whether the NCEP ATP III (HR:1.10, 95\% CI: 1.01 to 1.20 ), or the IDF definition (1.09 (1.01 to $1.19)$ ). For CVD mortality, MetS had a significant association using the IDF definition only (1.29 (1.05 to 1.57$)$ ) after adjusting for age and sex. ${ }^{37}$

Data on MetS-related mortality in Asian countries are still scarce. In an earlier study in Hong Kong, which involved a population-based cohort of 2863 Chinese subjects, those with MetS (revised NCEP-ATP III definition) had increased risk of all-cause (2.02 (1.02 to 4.0)) and CVD mortality (6.39 (1.40 to 29.2)) compared with those without any component of MetS controlling for baseline sociodemographic and lifestyle factors. However, the risk of death due to CVD in the MetS group was lower (3.36 (1.57 to 7.19)) compared with those with $\leq 2$ components, and no significant risk of death from all-causes was observed.$^{38}$ Our findings were also in line with a study conducted in Singapore. Using data from three cross-sectional surveys as baseline, a cohort of 5699 Singaporean adults from different ethnic groups was followed up for an average of 14 years. Those with metabolic syndrome (modified revised NCEP-ATP III) were found to have had an increased risk of death from both all-cause (men:1.4 (1.1-1.8), women: 1.8 (1.3-2.6)) and CVD mortalities (men: 3.0 (1.9-4.8), women: $2.1(1.1-4.0)$ ) after controlling for age, study, ethnic group, LDL-C, smoking and alcohol intake. ${ }^{39}$ A prospective study among 96433 middle aged and elderly Japanese adults who participated in a large community survey also reported an association between MetS and excess CVD deaths regardless of BMI status (overweight or non-overweight), sex and the MetS definition used. ${ }^{40}$ In Taiwan, a cohort which consisted of 10547 elderly (aged 65 years and older) individuals from nationwide health screenings between 1998 and 1999 were recruited to investigate if there was an association between the presence of MetS and risk cardiovascular mortality. ${ }^{41}$ After a follow-up of 8 years, those with MetS (revised NCEP-ATP III criteria) had significantly increased risk of CVD mortality (1.48 (1.16-1.90)). However, the strength of the association was relatively small for all-cause mortality (1.16 (1.03-1.30)) and possibly inconsequential in predicting all-cause mortality in clinical practice. This could be due to the study samples being composed entirely of the elderly, who were equally more prone to other causes of death, which reduces the relative effect of MetS. ${ }^{37}$ In comparison to our findings, a large retrospective study in Korea showed an insignificant association between MetS and all-cause mortality (1.15 (0.94-1.42)), and a significant but weaker association with CVD mortality (1.60 (1.02-2.20)), after adjusting for age, sex, smoking status, alcohol intake and regular exercise. ${ }^{42}$ But the shorter follow-up period of 3.7 years, compared with the 13 years follow-up in our study, may have not been sufficient to observe long-term effects of MetS on risk of all-cause mortality. ${ }^{43}$ In a recent Iranian study involving a sample of 523 healthy and $502 \mathrm{MetS}$ adults from the Zanjan Healthy Heart Study 2013. After a follow-up of approximately 10 years, the study revealed that risk of death from CVD events (3.7 (1.2-11.1)) was significantly higher among the MetS group than the healthy group. However, no significant increase in risk of all-cause mortality (1.75 (0.883.47)) was observed. ${ }^{44}$ This is possibly because of their high drop-out rate, resulting in small sample size and insufficient power to detect an association.

Our findings add to the existing evidence on the association of MetS with all-cause and CVD mortality in Asian populations. The HRs found in this study were comparable to studies in Western populations and some Asian countries, bearing in mind possible differences in MetS definition, age and race composition between countries. Our study has several strengths and limitations. A particular strength of this study was the data came from a nationally representative, population-based study and with a large sample size, which makes the findings generalisable to the non-institutionalised population of Malaysia. We also controlled for potential confounders including age, gender, physical activity, fruits and vegetables intake, smoking and alcohol consumption ${ }^{45-48}$ which, in Cox regression models, might undermine the relationship between MetS and risk of death. One of the limitations of this study was we cannot rule out the possibility of patients having undiagnosed cancer or other debilitating illness at baseline. Therefore, a sensitivity analysis was also conducted to assess this association, whereby participants who died in the first 2 years of the study were excluded. An additional limitation is the inability to incorporate time-varying covariates in the Cox model because information on transition from non-MetS to MetS is not available. Finally, there were deaths that were not medically certified. In these cases, CVD deaths may have been misclassified as deaths due to other poorly defined causes such as 'old age' or 'sudden death', consequently leading to underestimation of CVD mortality. ${ }^{49}$ However, medical certification of deaths in Malaysia has significantly improved over the years. In 1995, the percentage of medically certified deaths was $45 \%$ of total deaths, but efforts are constantly being made for improving the death reporting system and by 2010 , the rate of medically certified deaths had risen to $57 \% .^{50}$

\section{CONCLUSION}

Our study showed that metabolic syndrome is associated with increased hazard of all-cause and CVD mortality in the multiethnic Malaysian population, confirming the findings of earlier studies in other populations. Therefore, lifestyle modification and pharmacological treatment approaches might improve the individual components forming the MetS, and thereby reduce mortalities associated with MetS. 


\section{Author affiliations}

${ }^{1}$ Sector for Biostatistics \& Data Repository, National Institutes of Health, Shah Alam, Selangor, Malaysia

${ }^{2}$ Institute for Medical Research, Setia Alam, Shah Alam, Selangor, Malaysia

${ }^{3}$ Universiti Utara Malaysia, Sintok, Kedah, Malaysia

${ }^{4}$ Centre for Nutrition Epidemiology Research, Institute for Public Health, Kuala Lumpur, Wilayah Persekutuan, Malaysia

${ }^{5}$ Hospital Sultan Ismail, Johor Bahru, Johor Darul Ta'zim, Malaysia

${ }^{6}$ NCD Section, Disease Control Division, Ministry of Health Malaysia, Putrajaya,

Wilayah Persekutuan, Malaysia

Acknowledgements The authors express their gratitude to the Director-General of Health, Malaysia for granting permission to publish this paper; the Manager of the National Institutes Health for support and the Non-Communicable Disease Section, Disease Control Division, Ministry of Health, Putrajaya for providing data from the Malaysia NCD Surveillance-1, MOH. The authors also thank the Malaysian National Registration Department for providing mortality data for this study.

Contributors KCC, KHL, SMG, CHT, AMAH, AB and HLL involved in the data management, statistical analysis and preparation of the manuscript. YKC, FIM and MAO provided the critical review of the manuscript. All authors read and approved the final manuscript.

Funding This study was supported by the National Institutes of Health, Ministry of Health Malaysia (NMRR-20-772-52989).

Disclaimer This research received no specific grant from any funding agency in the public, commercial or not-for-profit sectors.

Competing interests None declared.

Patient consent for publication Not required.

Ethics approval The Malaysian Medical Research and Ethics Committee provided the ethical approval for this study (NMRR-20-772-52989).

Provenance and peer review Not commissioned; externally peer reviewed.

Data availability statement Data are available upon reasonable request. The data are not publicly available due to the personal privacy of subjects but are available from the corresponding author on reasonable request.

Supplemental material This content has been supplied by the author(s). It has not been vetted by BMJ Publishing Group Limited (BMJ) and may not have been peer-reviewed. Any opinions or recommendations discussed are solely those of the author(s) and are not endorsed by BMJ. BMJ disclaims all liability and responsibility arising from any reliance placed on the content. Where the content includes any translated material, BMJ does not warrant the accuracy and reliability of the translations (including but not limited to local regulations, clinical guidelines, terminology, drug names and drug dosages), and is not responsible for any error and/or omissions arising from translation and adaptation or otherwise.

Open access This is an open access article distributed in accordance with the Creative Commons Attribution Non Commercial (CC BY-NC 4.0) license, which permits others to distribute, remix, adapt, build upon this work non-commercially, and license their derivative works on different terms, provided the original work is properly cited, appropriate credit is given, any changes made indicated, and the use is non-commercial. See: http://creativecommons.org/licenses/by-nc/4.0/.

\section{ORCID iDs}

Kee Chee Cheong http://orcid.org/0000-0003-1372-9549

Kuang Hock Lim http://orcid.org/0000-0001-9364-2536

\section{REFERENCES}

1 Watanabe J, Kakehi E, Kotani K, et al. Metabolic syndrome is a risk factor for cancer mortality in the general Japanese population: the Jichi medical school cohort study. Diabetol Metab Syndr 2019:11:1-12.

2 Shi TH, Wang B, Natarajan S. The influence of metabolic syndrome in predicting mortality risk among US adults: importance of metabolic syndrome even in adults with normal weight. Prev Chronic Dis 2020;17:1-10.

3 Käräjämäki AJ, Bloigu R, Kauma $\mathrm{H}$, et al. Non-alcoholic fatty liver disease with and without metabolic syndrome: different long-term outcomes. Metabolism 2017;66:55-63.

4 Lorenzo C, Williams K, Hunt KJ, et al. The National Cholesterol Education Program - Adult Treatment Panel III, International Diabetes
Federation, and World Health Organization definitions of the metabolic syndrome as predictors of incident cardiovascular disease and diabetes. Diabetes Care 2007;30:8-13.

5 Gami AS, Witt BJ, Howard DE, et al. Metabolic syndrome and risk of incident cardiovascular events and death: a systematic review and meta-analysis of longitudinal studies. J Am Coll Cardiol 2007:49:403-14.

6 Mottillo S, Filion KB, Genest J, et al. The metabolic syndrome and cardiovascular risk a systematic review and meta-analysis. J Am Coll Cardiol 2010;56:1113-32.

7 Eckel RH, Grundy SM, Zimmet PZ. The metabolic syndrome. Lancet 2005;365:1415-28

8 Ranasinghe P, Mathangasinghe $\mathrm{Y}$, Jayawardena R, et al. Prevalence and trends of metabolic syndrome among adults in the Asia-Pacific region: a systematic review. BMC Public Health 2017;17:1-9.

9 Hoang KC, Le TV, Wong ND. The metabolic syndrome in East Asians. J Cardiometab Syndr 2007;2:276-82.

10 Misra A, Wasir JS, Vikram NK. Waist circumference criteria for the diagnosis of abdominal obesity are not applicable uniformly to all populations and ethnic groups. Nutrition 2005;21:969-76.

11 World Health Organization Regional Office for the Western Pacific, International Diabetes Institute, International Association for the Study of Obesity IOTF. The Asia-Pacific perspective: redefining obesity and its treatment, 2000. Available: https://iris.wpro.who.int/ handle/10665.1/5379

12 Lear SA, Humphries KH, Kohli S, et al. The use of BMI and waist circumference as surrogates of body fat differs by ethnicity. Obesity 2007:15:2817-24.

13 Malik S, Wong ND, Franklin SS, et al. Impact of the metabolic syndrome on mortality from coronary heart disease, cardiovascular disease, and all causes in United States adults. Circulation 2004; 110:1245-50.

14 Eberly LE, Prineas R, Cohen JD, et al. Metabolic syndrome: risk factor distribution and 18-year mortality in the multiple risk factor intervention trial. Diabetes Care 2006;29:123-30.

15 Liu L, Miura K, Fujiyoshi A, et al. Impact of metabolic syndrome on the risk of cardiovascular disease mortality in the United States and in Japan. Am J Cardiol 2014;113:84-9.

16 Department of Statistics Malaysia. Statistics on causes of death, Malaysia, 2020. Available: https://www.dosm.gov.my/v1/index.php? $r=$ column/cthemeByCat\&cat=401\&bul id=QTU5T0dKQ1g4MHYx d3ZpMzhEMzdRdz09\&menu_id=LOpheU43NWJwRWVSZkIWdzQ4 TIhUUT09 [Accessed 21 May 2021].

17 Ismail M. CI. T. WHO/IASO/IOTF. The Asia-Pacific perspective: redefining obesity and its treatment. Health Communications Australia: Melbourne, 2000

18 Tan BY, Kumar Kantilal H, Singh R. Prevalence of metabolic syndrome among Malaysians using the International diabetes Federation, National cholesterol education program and modified World Health organization definitions. World Health Organization, 2008.

19 Termizy HM, Mafauzy M. Metabolic syndrome and its characteristics among obese patients attending an obesity clinic. Singapore Med J 2009:50:390-4

20 Moy FM, Bulgiba A. The modified NCEP ATP III criteria maybe better than the IDF criteria in diagnosing metabolic syndrome among Malays in Kuala Lumpur. BMC Public Health 2010;10:1-6.

21 Mohamud WNW, Ismail Aal-S, Sharifuddin A, et al. Prevalence of metabolic syndrome and its risk factors in adult Malaysians: results of a nationwide survey. Diabetes Res Clin Pract 2011;91:239-45.

22 Ministry of Health Malaysia. Malaysia NCD surveillance 2006 report NCD risk factors in Malaysia. Minist Heal Malaysia, 2006: 1-133.

23 Dupont WD, Plummer WD. Power and sample size calculations for studies involving linear regression. Control Clin Trials 1998;19:589-601.

24 World Health Organization. Guidelines for controlling and monitoring the tobacco epidemic. Available: https://apps.who.int/iris/ bitstream/handle/10665/42049/9241545089-eng.pdf?sequence=8\& isAllowed=y [Accessed 2 Jun 2021].

25 World Health Organization. Stepwise approach to ncd risk factor surveillance (steps). Available: https://www.who.int/teams/ noncommunicable-diseases/surveillance/systems-tools/steps [Accessed 2 Jun 2021].

26 World Health Organization. Non communicable diseases and their risk factors: Global Physical Activity Surveillance. WHO, 2017. Available: http://www.who.int/ncds/surveillance/steps/GPAQ/en/ [Accessed 2 Jun 2021].

27 Expert Panel on Detection, Evaluation, and Treatment of High Blood Cholesterol in Adults. Executive summary of the third report of the National cholesterol education program (NCEP) expert panel on detection, evaluation, and treatment of high blood 
cholesterol in adults (adult treatment panel III). J Am Med Assoc 2001;285:2486-97.

28 Grundy SM, Cleeman JI, Daniels SR, et al. Diagnosis and management of the metabolic syndrome: an American heart Association/National heart, lung, and blood Institute scientific statement. Circulation 2005;112:2735-52.

29 Alberti KGMM, Zimmet P, Shaw J. Metabolic syndrome--a new world-wide definition. A Consensus Statement from the International Diabetes Federation. Diabet Med 2006;23:469-80.

30 Alberti KGMM, Eckel RH, Grundy SM, et al. Harmonizing the metabolic syndrome: a joint interim statement of the International diabetes Federation Task force on epidemiology and prevention; National heart, lung, and blood Institute; American heart association; world heart Federation; international atherosclerosis Society; and international association for the study of obesity. Circulation 2009;120:1640-5.

31 Cheong KC, Ghazali SM, Hock LK, et al. Optimal waist circumference cut-off values for predicting cardiovascular risk factors in a multiethnic Malaysian population. Obes Res Clin Pract 2014;8:e154-62.

32 Ramli AS, Daher AM, Nor-Ashikin MNK, et al. JIS definition identified more Malaysian adults with metabolic syndrome compared to the NCEP-ATP III and IDF criteria. Biomed Res Int 2013;2013:1-10.

33 World Health Organization. International statistical classification of diseases and related health problems 10th revision (ICD-10), 2019. Available: https://icd.who.int/browse10/2019/en [Accessed 2 Jun 2021].

34 Lim KG, Cheah WK. A review of metabolic syndrome research in Malaysia. Med J Malaysia 2016;71:20-8

35 Krakauer NY, Krakauer JC. Anthropometrics, metabolic syndrome, and mortality hazard. J Obes 2018;2018:1-7.

36 Wu SH, Hui WS, Liu Z, et al. Metabolic syndrome and all-cause mortality: a meta-analysis of prospective cohort studies. Eur $\mathrm{J}$ Epidemiol 2010;25:375-84.

37 van Herpt TTW, Dehghan A, van Hoek M, et al. The clinical value of metabolic syndrome and risks of cardiometabolic events and mortality in the elderly: the Rotterdam study. Cardiovasc Diabetol 2016:15:1-10.

38 Thomas GN, Schooling CM, McGhee SM, et al. Metabolic syndrome increases all-cause and vascular mortality: the Hong Kong cardiovascular risk factor study. Clin Endocrinol 2007;66:666-71.
39 Lee J, Heng D, Ma S, et al. The metabolic syndrome and mortality: the Singapore cardiovascular cohort study. Clin Endocrinol 2008;69:225-30.

40 Irie $\mathrm{F}$, Iso $\mathrm{H}$, Noda $\mathrm{H}$, et al. Associations between metabolic syndrome and mortality from cardiovascular disease in Japanese general population, findings on overweight and non-overweight individuals. Ibaraki Prefectural health study. Circ J 2009;73:1635-42.

41 Wen CJ, Lee YS, Lin WY, et al. The metabolic syndrome increases cardiovascular mortality in Taiwanese elderly. Eur J Clin Invest 2008;38:469-75.

42 Sung K-C, Rhee E-J, Ryu S, et al. Increased cardiovascular mortality in subjects with metabolic syndrome is largely attributable to diabetes and hypertension in 159,971 Korean adults. J Clin Endocrinol Metab 2015;100:2606-12.

43 Meinow B, Kåreholt I, Parker MG, et al. The effect of the duration of follow-up in mortality analysis: the temporal pattern of different predictors. J Gerontol B Psychol Sci Soc Sci 2004;59:S181-9.

44 Mazloomzadeh S, Zarandi FK, Shoghli A. Metabolic syndrome, its components and mortality: a population-based study. Med J Islam Repub Iran 2019;33:60-4.

45 Moe B, Mork PJ, Holtermann A, et al. Occupational physical activity, metabolic syndrome and risk of death from all causes and cardiovascular disease in the HUNT 2 cohort study. Occup Environ Med 2013;70:86-90.

46 Takashima N, Miura K, Hozawa A, et al. Population attributable fraction of smoking and metabolic syndrome on cardiovascular disease mortality in Japan: a 15-year follow up of nippon DATA90. BMC Public Health 2010;10:306.

47 Hildrum B, Mykletun A, Dahl AA, et al. Metabolic syndrome and risk of mortality in middle-aged versus elderly individuals: the NordTrøndelag health study (Hunt). Diabetologia 2009;52:583-90.

48 Xi B, Veeranki SP, Zhao M, et al. Relationship of alcohol consumption to all-cause, cardiovascular, and cancer-related mortality in U.S. adults. J Am Coll Cardiol 2017;70:913-22.

49 Faudzi AY, Amal NM, Zainal AO, et al. Improvement in medically certified death: results from a pilot study in the state of malacca, Malaysia. Med J Malaysia 2011;66:32-5.

50 Adnan TH, Bujang MA, Supramaniam P. Trend Analysis of Medically Certified Death in Malaysia, 1995 - 2010. J Health Inform Dev Ctries 2012;6:396-405. 